\title{
INFINITE DIMENSIONAL ANALYSIS OF PURE JUMP LÉVY PROCESSES ON THE POISSON SPACE
}

\author{
ARNE LØKKA and FRANK NORBERT PROSKE*
}

\begin{abstract}
We develop a white noise calculus for pure jump Lévy processes on the Poisson space. This theory covers the treatment of Lévy processes of unbounded variation. The starting point of the theory is the construction of a distribution space. This space has many of the same nice properties as the classical Schwartz space, but is modified in a certain way in order to be more suitable for pure jump Lévy processes. We apply Minlos's theorem to this space and obtain a white noise measure which satisfies the first condition of analyticity, and which is non-degenerate. Furthermore, we obtain generalized Charlier polynomials for all Lévy measures. We introduce Kondratiev test function and distribution spaces, the $\mathscr{S}$-transform and the Wick product. We proceed by using a transfer principle on Poisson spaces to establish a differential calculus.
\end{abstract}

\section{Introduction}

The main objective of this paper is to construct a suitable white noise framework for pure jump Lévy processes. There are several papers dealing with white noise analysis and pure jump Lévy processes (see, e.g., [14], [13]), but to the best of our knowledge, none of these present a framework that is suitable for all pure jump Lévy processes. Some restrictions, typically integrability conditions, are put on the Lévy measure. This paper presents a framework which works for all pure jump Lévy processes.

A pure jump Lévy process $L$ with no drift, is a martingale with independent and stationary increments, continuous in probability and with no Brownian motion part. The Lévy-Khintchine formula provides an expression for the characteristic function of such a process in terms of a measure $v$, called the Lévy measure of the Lévy process. Thus, pure jump Lévy processes with no drift can be characterized as Lévy processes with characteristic triplet $(0,0, v)$ (see, e.g., [18] for more details). The Poisson space is a natural space for dealing with Lévy processes for several reasons. One of them is that Lévy processes,

\footnotetext{
${ }^{*}$ We would like to thank F. E. Benth, J. L. Da Silva, B. Øksendal, J. Potthoff, T. Zhang and an anonymous referee for fruitful discussions and helpful comments. Arne Løkka acknowledges the financial support from the Norwegian Research Council, grant 134228/432.

Received December 11, 2003; in revised form January 13, 2005.
} 
in general, do not possess the chaos representation property with respect to the Lévy process itself. However, every square integrable functional of the path of a pure jump Lévy process, has according to [9], a chaos representation with respect to Poisson random measures. By viewing a Lévy process as an element in an appropriate Poisson space, we therefore obtain a more unified and tractable framework. In addition, every functional on the Poisson space has a chaos expansion in terms of generalized Charlier polynomials.

There are several papers on infinite dimensional analysis on the Poisson space, with varying degrees of generality regarding the intensity measure. However, as far as the authors know, the existing literature contain no explicit construction which works for intensities that are the product of the Lebesgue measure and an arbitrary Lévy measure. A usual starting point in white noise analysis is to apply the Bochner-Minlos theorem, which guarantees the existence of a probability measure on the space of tempered distributions $\mathscr{S}^{\prime}\left(\mathrm{R}^{d}\right)$. For instance [11] deals with infinite dimensional analysis for Poisson measures based on the classical Schwartz space. However, it turns out that the classical Schwartz space $\mathscr{S}^{\prime}\left(\mathrm{R}^{d}\right)$ is not the most appropriate distribution space for dealing with arbitrary pure jump Lévy processes on the Poisson space, since this choice requires restrictive conditions to be put on the Lévy measure. This is due to the fact that the Lévy measure, in general, has a singularity at zero. Therefore, the corresponding characteristic Poisson functional is not necessarily continuous on $\mathscr{S}\left(\mathrm{R}^{d}\right)$. Section two is devoted to the construction of a nuclear algebra $\widetilde{\mathscr{S}}(X)$, which is a variation of the Schwartz space on the space $X$, but which is more suitable for our purpose. Within this framework, we show that any Lévy measure has a Radon-Nikodym derivative with respect to the Lebesgue measure in a generalized sense. We denote this derivative by $\dot{v}$. The Bochner-Minlos theorem is then used to prove the existence of a probability measure $\mu_{\pi}$, with Poissonian characteristic functional with intensity of the form $\pi_{v}=\lambda^{\times d} \times v(\lambda$ being the Lebesgue measure $)$, such that

$$
\int_{\widetilde{\mathscr{P}}^{\prime}(X)} e^{i\langle\omega, \phi\rangle} d \mu_{\pi}(\omega)=\exp \left(\int_{X}\left(e^{i \phi(x)}-1\right) \pi(d x)\right),
$$

for all $\phi \in \tilde{\mathscr{S}}(X)$. The continuity of the functional on the right hand side of (1) follows from the existence of a generalized Radon-Nikodym derivative of the Lévy measure with respect to the Lebesgue measure. By using an idea of Us [21], we can prove that $\mu_{\pi}$ satisfies the first condition of analyticity (See Lemma 2.6). Furthermore, we show that the measure $\mu_{\pi}$ is non-degenerate in the sense of [12]. Roughly speaking the non-degeneracy is due to the fact that in the construction of $\mathscr{\mathscr { S }}(X)$, we have identified all functions which are equal on $\pi_{v}$-null sets. We then have all we need in order to have a well defined 
system of orthogonal generalized Charlier polynomials. The construction and existence of such polynomials is the topic of section 3 and is similar to the constructions in [1] and [13].

We proceed in section 4 by extending the chaos expansion in terms of Charlier polynomials. This is done by a construction of stochastic test functions and stochastic distributions of the Kondratiev type. Our construction is a variation of the construction in [11] and corresponds to the $(\mathscr{S}(J))^{-1}$ distribution space in [21].

In section 5 we define the $\mathscr{S}$-transform and the Wick product. This is included since we believe that the $\mathscr{S}$-transform and the Wick product are useful mathematical tools. It was proved in [1] that the image of the Kondratiev distributions under the $\mathscr{S}$-transform is equal to a certain space of holomorphic functionals. This characterization is valid also in our case, and we can define the Wick product of two distributions as the inverse image of the $\mathscr{S}$-transform of the product of the $\mathscr{S}$-transform of the distributions.

The measure $\mu_{\pi}$ does not admit a satisfactory construction of a differential calculus on $\widetilde{\mathscr{S}}^{\prime}(X)$. In section 6 we show how we can circumvent this problem by transporting analytical structures from configuration spaces by using a unitary isomorphism in a similar fashion as in [13] and [11]. This yields a Poisson measure $\mu_{\pi}^{\Gamma}$ on the configuration space $(\Gamma, \mathscr{B}(\Gamma))$, such that $L^{2}\left(\Gamma, \mu_{\pi}^{\Gamma}\right)$ is unitary isomorphic to $L^{2}\left(\mu_{\pi}\right)$.

Section 7 deals with the Poissonian gradient and Skorohod integration. We start by proving that the Poissonian gradient $\nabla^{P}$ and the operator $D$ defined by its action on chaos expansions in terms of Charlier polynomials, essentially are equal. The Skorohod integral is then defined by its action on the chaos expansions of parametrized families of stochastic distributions. We show that Skorohod integration is the dual of the Poissonian gradient $\nabla^{P}$. Hence, Skorohod integration is equal to $\left(\nabla^{P}\right)^{*}$ and we can link the Skorohod integral with results for $\left(\nabla^{P}\right)^{*}$ in [13]. These results can be viewed as generalizations of some of the results in [17]. By using the duality between $\nabla^{P}$ and the Skorohod integral, we can also prove relations between the $\mathscr{S}$-transform, ordinary derivation and the Skorohod integral. Finally, we prove a relationship between the Skorohod integral, the Wick product and the Lebesgue integral.

\section{Construction of the probability space}

We start with a review of some well known facts about the classical Schwartz space. Let $\xi_{n}$ denote the $n$ 'th Hermite function. The set of Hermite functions $\left\{\xi_{n}\right\}_{n \in \mathrm{N}}$ is an orthonormal basis for $L^{2}(\mathrm{R})$. The (countably Hilbertian) nuclear topology on the classical Schwartz space $\mathscr{S}\left(\mathrm{R}^{d}\right)$ is induced by the compatible 
system of norms

$$
\|\phi\|_{\beta}^{2}:=\sum_{\alpha \in \mathbb{N}^{d}}(1+\alpha)^{2 \beta}\left(\phi, \xi_{\alpha}\right)_{L^{2}\left(\mathbb{R}^{d}\right)}^{2},
$$

for $\alpha=\left(\alpha_{1}, \ldots, \alpha_{d}\right) \in \mathrm{N}^{d}$ and $\beta=\left(\beta_{1}, \ldots, \beta_{d}\right) \in \mathbf{N}_{0}^{d}$, where

$$
\xi_{\alpha}:=\prod_{i=1}^{d} \xi_{\alpha_{i}}, \quad(1+\alpha)^{2 \beta}:=\prod_{i=1}^{d}\left(1+\alpha_{i}\right)^{2 \beta_{i}} .
$$

The topology on $\mathscr{S}\left(\mathrm{R}^{d}\right)$ is also induced by the smaller system of pre-Hilbertian norms

$$
\|\cdot\|_{p}:=\|\cdot\|_{\beta}, \quad p \in \mathrm{N}_{0}, \quad \beta=(p, \ldots, p) \in \mathrm{N}_{0}^{d} .
$$

It is well known (see, e.g., [19]) that the system of norms $\|\cdot\|_{p}$ is equivalent to the system of norms

$$
\|\phi\|_{q, \infty}:=\sup _{\alpha \geq 0,|\beta| \leq q} \sup _{x \in \mathbb{R}^{d}}\left|\left(1+|x|^{\alpha}\right)\left(D^{\beta} \phi\right)(x)\right|, \quad q \in \mathbf{N}_{0},
$$

for $\beta=\left(\beta_{1}, \ldots, \beta_{d}\right) \in \mathrm{N}_{0}^{d}$, where $|\beta|:=\beta_{1}+\cdots+\beta_{d}$ and

$$
D^{\beta}:=\frac{\partial^{|\beta|}}{\partial x_{1}^{\beta_{1}} \cdots \partial x_{d}^{\beta_{d}}} .
$$

An important property of $\|\cdot\|_{p}$ is that for every $p \in \mathrm{N}_{0}$ there exists a constant $M_{p}$, such that for every $\phi, \psi \in \mathscr{S}\left(\mathrm{R}^{d}\right)$,

$$
\|\phi \psi\|_{p} \leq M_{p}\|\phi\|_{p}\|\psi\|_{p}
$$

(see, e.g., [10]). It follows from (2.1) that the Schwartz space $\mathscr{S}\left(\mathrm{R}^{d}\right)$ is not only a nuclear space, but also a nuclear algebra.

Set $X:=\mathrm{R}^{d} \times \mathrm{R}_{0}$, where $\mathrm{R}_{0}:=\mathrm{R} \backslash\{0\}$. Define the space

$$
\mathscr{S}(X):=\left\{\phi \in \mathscr{S}\left(\mathrm{R}^{d+1}\right): \phi\left(x_{1}, \ldots, x_{d}, 0\right)=\frac{\partial \phi}{\partial x_{d+1}}\left(x_{1}, \ldots, x_{d}, 0\right)=0\right\} .
$$

It follows that $\mathscr{S}(X)$ is a closed subspace of $\mathscr{S}\left(\mathrm{R}^{d+1}\right)$. Thus, $\mathscr{S}(X)$ is a countably Hilbertian nuclear algebra endowed with the topology induced by the norms $\|\cdot\|_{p}$. Moreover, let $\mathscr{S}^{\prime}(X)$ denote the dual of $\mathscr{S}(X)$. Since $\mathscr{S}(X) \subset$ $\mathscr{S}\left(\mathrm{R}^{d+1}\right)$, it follows that we have the inclusion $\mathscr{S}^{\prime}\left(\mathrm{R}^{d+1}\right) \subset \mathscr{S}^{\prime}(X)$. For $\Phi \in \mathscr{S}^{\prime}(X)$ and $\phi \in \mathscr{S}(X)$, the action $\Phi(\phi)$ is given by

$$
\Phi(\phi):=\langle\Phi, \phi\rangle:=\int_{X} \Phi(x) \phi(x) d \lambda^{\times(d+1)}(x),
$$


where $\lambda^{\times d}$ denote the Lebesgue measure on $\mathrm{R}^{d}$. In the sequel, we will consequently let $v$ denote a Lévy measure on $\mathbf{R}_{0}$ and let $\pi_{v}$ denote the measure on $X$ given by $\pi_{v}:=\lambda^{\times d} \times \nu$. When there is no ambiguity, we will frequently write $\pi$ instead of $\pi_{\nu}$.

Lemma 2.1. For every Lévy measure $v$ there exists an element, denoted by $1 \otimes \dot{v}$, in $\mathscr{S}^{\prime}(X)$ such that

$$
\langle 1 \otimes \dot{v}, \phi\rangle=\int_{X} \phi(x) \pi_{v}(d x),
$$

for all $\phi \in \mathscr{S}(X)$.

Proof. Without loss of generality, we consider the case $d=1$. Set $L(\phi)=$ $\int_{X} \phi(x) \pi_{v}(d x)$. If we can prove that $L$ is a continuous linear functional on $\mathscr{S}(X)$, then $L \in \mathscr{S}^{\prime}(X)$ and the result follows. First note that $L$ is linear. We want to prove that $L$ is continuous. For every $\psi \in \mathscr{S}(X)$, it follows from Taylor's formula that

$$
\begin{aligned}
\psi(y, z) & =\psi(y, 0)+\frac{\partial \psi}{\partial z}(y, 0) \cdot z+\frac{1}{2} \frac{\partial^{2} \psi}{\partial z^{2}}(y, \xi) \cdot z^{2} \\
& =\frac{1}{2} \frac{\partial^{2} \psi}{\partial z^{2}}(y, \xi) \cdot z^{2},
\end{aligned}
$$

for every $y \in \mathbf{R}, z \in \mathbf{R}_{0}$ and some $\xi=\xi(y, z) \in \mathbf{R}_{0}$ between 0 and $z$. Now let $\left\{\phi_{n}\right\}_{n \in \mathrm{N}}$ be a sequence of functions in $\mathscr{S}(X)$ converging to $\phi \in \mathscr{S}(X)$ with respect to the topology of $\mathscr{S}(X)$. From (2.2) and the fact that $\left\|\phi_{n}-\phi\right\|_{q, \infty} \rightarrow 0$ as $n \rightarrow \infty$,

$$
\begin{aligned}
\left|L\left(\phi_{n}\right)-L(\phi)\right| & =\left|L\left(\phi_{n}-\phi\right)\right| \\
& \leq \int_{\mathrm{R}} \int_{\mathrm{R}_{0}}\left|\phi_{n}(y, z)-\phi(y, z)\right| v(d z) \lambda(d y) \\
& =\int_{\mathrm{R}} \int_{\mathrm{R}_{0}} \frac{1}{2} z^{2}\left|\frac{\partial^{2}\left(\phi_{n}-\phi\right)}{\partial \xi^{2}}(y, \xi(y, z))\right| v(d z) \lambda(d y) \\
& =\frac{1}{2} \int_{\mathrm{R}} \int_{\mathrm{R}_{0}}\left|\left(1+y^{2}+\xi^{2}(y, z)\right) \frac{\partial^{2}\left(\phi_{n}-\phi\right)}{\partial \xi^{2}}(y, \xi(y, z))\right| \\
& \times \frac{z^{2}}{1+y^{2}+\xi^{2}(y, z)} v(d z) \lambda(d y) \\
\leq & \frac{1}{2}\left\|\phi_{n}-\phi\right\|_{2, \infty} \int_{\mathrm{R}} \int_{\mathrm{R}_{0}} \frac{z^{2}}{1+y^{2}+\xi^{2}(y, z)} v(d z) \lambda(d y)
\end{aligned}
$$




$$
\leq \frac{1}{2}\left\|\phi_{n}-\phi\right\|_{2, \infty} \int_{\mathrm{R}} \int_{\mathrm{R}_{0}} \frac{z^{2}}{1+y^{2}} v(d z) \lambda(d y) \longrightarrow 0
$$

as $n \rightarrow \infty$ since $\int_{\mathrm{R}_{0}} \frac{z^{2}}{1+z^{2}} v(d z)<\infty$, for every Lévy measure $v$. From (2.3), it follows that $L$ is continuous on $\mathscr{S}(X)$.

We use the notation $1 \otimes \dot{v}$ in order to indicate that $\dot{v}$ is, in a generalized sense, the Radon-Nikodym derivative of $\pi_{v}$ with respect to the Lebesgue measure. Moreover, $\dot{v}$ is, in a generalized sense, the Radon-Nikodym derivative of $v$ with respect to Lebesgue measure.

Denote by $L^{2}\left(X^{n}, \pi^{\times n}\right)$ the space of all functions on $X^{n}$ which are square integrable with respect to $\pi^{\times n}$. Let $(\cdot, \cdot)_{\pi}$ denote the inner product on $L^{2}(X, \pi)$ and $|\cdot|_{\pi}$ the corresponding norm on this space.

Define a space $\mathcal{N}_{\pi}$ by

$$
\mathcal{N}_{\pi}:=\left\{\phi \in \mathscr{S}(X):|\phi|_{\pi}=0\right\} .
$$

By the same arguments as in the proof of Lemma 2.1, one can show that $\mathscr{N}_{\pi}$ is a closed ideal of $\mathscr{S}(X)$. Introduce another space denoted by $\widetilde{\mathscr{S}}(X)$. This space will be our starting point for the construction of the white noise measure.

Definition 2.2. We denote by $\tilde{\mathscr{S}}(X)$ the space

$$
\tilde{\mathscr{S}}(X):=\mathscr{S}(X) / \mathcal{N}_{\pi},
$$

endowed with the topology induced by the family of norms

$$
\|\hat{\phi}\|_{p, \pi}:=\inf _{\psi \in \mathcal{N}_{\pi}}\|\phi+\psi\|_{p} .
$$

The following result states that $\tilde{\mathscr{S}}(X)$ have similar nice properties as the classical Schwartz space.

THEOREM 2.3. The space $\tilde{\mathscr{S}}(X)$ is a nuclear algebra with a compatible system of norms given by (2.4). Moreover the Cauchy-Bunjakowski inequality holds, that is for all $p \in \mathrm{N}$ there exists an $M_{p}$ such that for all $\phi, \psi \in \widetilde{\mathscr{S}}(X)$ we have

$$
\|\phi \psi\|_{p, \pi} \leq M_{p}\|\phi\|_{p, \pi}\|\psi\|_{p, \pi} .
$$

Proof. It follows from [7, p. 72] that $\tilde{\mathscr{S}}(X)$ is a nuclear space with a compatible system of norms given by (2.4). Thus the only thing left to prove is the Cauchy-Bunjakowski inequality. First, note that $\mathscr{S}(X)$ is a nuclear algebra which satisfies (2.1). Now, choose $\phi, \psi \in \mathscr{S}(X)$ and $\rho_{1}, \rho_{2} \in \mathcal{N}_{\pi}$. Then,

$$
\left\|\left(\phi+\rho_{1}\right)\left(\psi+\rho_{2}\right)\right\|_{p} \leq M_{p}\left\|\phi+\rho_{1}\right\|_{p}\left\|\psi+\rho_{2}\right\|_{p}
$$


and

$$
\left\|\left(\phi+\rho_{1}\right)\left(\psi+\rho_{2}\right)\right\|_{p}=\left\|\phi \psi+\rho_{3}\right\|_{p} \geq\|\hat{\phi} \hat{\psi}\|_{p, \pi},
$$

where $\rho_{3} \in \mathcal{N}_{\pi}$. The result then follows from (2.5) and (2.6).

Let $\tilde{\mathscr{S}}^{\prime}(X)$ denote the topological dual of $\tilde{\mathscr{S}}(X)$, which is isomorphic to the orthogonal complement of $\mathcal{N}_{\pi}$ (see, e.g., [20]). For every $p \in \mathrm{N}$ let $\widetilde{\mathscr{S}}_{p}(X)$ denote the completion of $\tilde{\mathscr{S}}(X)$ with respect to the norm $\|\cdot\|_{p, \pi}$. Moreover, let $\widetilde{\mathscr{S}}_{-p}(X)$ denote the dual of $\widetilde{\mathscr{S}}_{p}(X)$.

Lemma 2.4. There exists a $p_{0} \in \mathrm{N}$ such that the functional $L(\hat{\phi}):=$ $\int_{X} \phi(x) \pi(d x)$ satisfies

$$
|L(\hat{\phi})| \leq M_{p}\|\hat{\phi}\|_{p, \pi}
$$

for all $p \geq p_{0}$. Thus, the functional $L$ is continuous on $\tilde{\mathscr{S}}(X)$.

Proof. Let $\psi \in \mathscr{N}_{\pi}$. It follows from Lemma 2.1 that there exists a $p_{0} \in \mathbf{N}$ such that

$$
|L(\hat{\phi})|=\left|\int_{X}(\phi+\psi)(x) \pi(d x)\right| \leq M_{p}\|\phi+\psi\|_{p},
$$

for all $p \geq p_{0}$. By taking the infimum over all $\psi \in \mathcal{N}_{\pi}$, the result follows.

THEOREM 2.5. There exists a probability measure $\mu_{\pi}$ on $\tilde{\mathscr{S}}^{\prime}(X)$ such that for all $\phi \in \widetilde{\mathscr{S}}(X)$,

$$
\int_{\tilde{\mathscr{S}}^{\prime}(X)} e^{i\langle\omega, \phi\rangle} d \mu_{\pi}(\omega)=\exp \left(\int_{X}\left(e^{i \phi}-1\right) d \pi\right) .
$$

Moreover, there exists a $p_{0} \in \mathrm{N}$ such that $1 \otimes \dot{v} \in \widetilde{\mathscr{S}}_{-p_{0}}(X)$, and a natural number $q_{0}>p_{0}$ such that the embedding operator $\tilde{\mathscr{S}}_{q_{0}}(X) \hookrightarrow \widetilde{\mathscr{S}}_{p_{0}}(X)$ is Hilbert-Schmidt and

$$
\mu_{\pi}\left(\widetilde{\mathscr{S}}_{-q_{0}}(X)\right)=1 .
$$

Proof. Consider the functional $\Phi$ given by

$$
\Phi(\phi):=\exp \left(\int_{X}\left(e^{i \phi(x)}-1\right) \pi(d x)\right)
$$

which is positive definite and satisfies $\Phi(0)=1$. Set $F(\phi)=\ln (\Phi(\phi))$. We have that

$$
\left|F(\phi)-F\left(\phi_{k}\right)\right|=\left|\int_{X}\left(e^{i \phi(x)}-1\right) \pi(d x)-\int_{X}\left(e^{i \phi_{k}(x)}-1\right) \pi(d x)\right|
$$




$$
\begin{aligned}
& =\left|\int_{X}\left(e^{i \phi(x)}-e^{i \phi_{k}(x)}\right) \pi(d x)\right| \\
& \leq \int_{X}\left|\phi(x)-\phi_{k}(x)\right| \pi(d x) .
\end{aligned}
$$

From (2.8) and Lemma 2.4, it follows that $F$, and hence $\Phi$, is continuous on $\widetilde{\mathscr{S}}(X)$. The result then follows from the Bochner-Minlos theorem for conuclear spaces (see, e.g., [8, Thm. 1.1, pp. 2]).

From now on, denote by $p_{0}$ and $q_{0}$ the numbers described in Theorem 2.5.

LEMMA 2.6 (1. condition of analyticity). $\mu_{\pi}$ satisfies the first condition of analyticity, that is there exists $\epsilon>0$ such that

$$
\int_{\tilde{\mathscr{S}}^{\prime}(X)} \exp \left(\epsilon\|\omega\|_{-q_{0}, \pi}\right) d \mu_{\pi}(\omega)<\infty
$$

Proof. The proof follows the argument of [21, Lemma 3]. Define,

$$
\Psi(\phi)=\exp \left(\int_{X}\left(e^{\phi(x)}-1\right) \pi(d x)\right),
$$

for $\phi \in \mathscr{S}(X)$. Introduce the moment functions of $\mu_{\pi}$, which by a criterion of Cramer [3] can be expressed by

$$
M_{n}(\phi):=\int_{\widetilde{\mathscr{S}}^{\prime}(X)}\langle\omega, \phi\rangle^{n} d \mu_{\pi}(\omega)=\left.\frac{d^{n} \Psi(t \phi)}{d t^{n}}\right|_{t=0},
$$

for every $\phi \in \tilde{\mathscr{S}}(X), t \in \mathrm{R}$ and $n \in \mathrm{N}$. Denote by $\Lambda_{n}^{k}$ the set

$$
\Lambda_{n}^{k}:=\left\{\left(\alpha_{1}, \ldots, \alpha_{k}\right) \in \mathrm{N}^{k}: \alpha_{i} \geq 1, \sum_{i=1}^{k} \alpha_{i}=n\right\} .
$$

We can then deduce the following expression for $M_{n}$,

$$
M_{n}(\phi)=\sum_{k=1}^{n} \frac{n !}{k !} \sum_{\alpha \in \Lambda_{n}^{k}} \prod_{j=1}^{k} \frac{\left\langle 1 \otimes \dot{v}, \phi^{\alpha_{j}}\right\rangle}{\alpha_{j} !} .
$$

Observe that

$$
|\langle 1 \otimes \dot{v}, \phi\rangle| \leq\|1 \otimes \dot{v}\|_{-q_{0}, \pi}\|\phi\|_{q_{0}, \pi}<\infty .
$$


By Theorem 2.3, there exists a constant $C_{q_{0}}$ such that

$$
\|\phi \psi\|_{q_{0}, \pi} \leq C_{q_{0}}\|\phi\|_{q_{0}, \pi}\|\psi\|_{q_{0}, \pi} \quad \text { for all } \quad \phi, \psi \in \tilde{\mathscr{S}}(X) .
$$

From (2.10), (2.11) and the expression for $M_{n}$ given by (2.9),

$$
\begin{aligned}
\left|M_{n}(\phi)\right| & \leq \sum_{k=1}^{n} \frac{n !}{k !} \sum_{\alpha \in \Lambda_{n}^{k}} \prod_{j=1}^{k} \frac{\|1 \otimes \dot{v}\|_{-q_{0}, \pi}\left\|\phi^{\alpha_{j}}\right\|_{q_{0}, \pi}}{\alpha_{j} !} \\
& \leq \sum_{k=1}^{n} \frac{n !}{k !} \sum_{\alpha \in \Lambda_{n}^{k}} \prod_{j=1}^{k} \frac{\|1 \otimes \dot{v}\|_{-q_{0}, \pi}}{\alpha_{j} !} C_{q_{0}}^{n}\|\phi\|_{q_{0}, \pi}^{n} \\
& =F_{n}\left(\|1 \otimes \dot{v}\|_{-q_{0}, \pi}\right) C_{q_{0}}^{n}\|\phi\|_{q_{0}, \pi}^{n},
\end{aligned}
$$

where $F_{n}(x)$ is the $n$ 'th moment of the Poisson distribution with intensity $x$. We know that for the Poisson distribution with intensity parameter $x=$ $\|1 \otimes \dot{v}\|_{-q_{0}, \pi}<\infty$ there exists a constant $C_{x}$ such that for all $n \in \mathrm{N}$,

$$
\left|F_{n}\left(\|1 \otimes \dot{v}\|_{-q_{0}, \pi}\right)\right| \leq n ! C_{\|1 \otimes \dot{v}\|_{-q_{0}, \pi}^{n}} .
$$

Hence there is a constant $C>0$, such that for all $n \in \mathrm{N}$ and $\phi \in \tilde{\mathscr{S}}(X)$ and

$$
\left|M_{n}(\phi)\right| \leq n ! C^{n}\|\phi\|_{q_{0}, \pi}^{n},
$$

from which the claimed result follows (see, [12, Lemma 3]).

Set $\Omega:=\tilde{\mathscr{S}}(X)$ and let $\mu_{\pi}$ be the measure given by Theorem 2.5. This provides us with a well defined Poisson white noise probability space that is suitable for an infinite dimensional calculus for every pure jump Lévy process.

\section{Chaos expansion and orthogonal polynomials}

In section 2 we constructed a probability measure $\mu_{\pi}$ on $\Omega=\tilde{\mathscr{S}}^{\prime}(X)$, which by Lemma 2.6 satisfies the first condition of analyticity. According to [1], this is all we need in order to have a chaos expansion of functionals in terms of Charlier polynomials. Our chaos expansion is a generalization of the chaos expansion in [13] to Poisson measures with a more general intensity measure than considered in [13].

Let $Y, Z$ be linear topological spaces. For a mapping $f: Y \rightarrow Z$, we define the directional derivative in the direction $\phi \in Y$ at the point $y \in Y$ by

$$
\nabla_{\phi} f(y)=\left.\frac{d}{d \epsilon} f(y+\epsilon \phi)\right|_{\epsilon=0} .
$$


We say that this mapping is differentiable along a subspace $\Lambda \subset Y$, if the derivative $\nabla_{\phi} f(y)$ exists for any $\phi \in \Lambda$ and $\nabla_{\phi} f(y)=f^{\prime}(y) \phi$, where $f^{\prime}(y)$ is a linear continuous mapping of $\Lambda$ into $Y$. Let $\nabla$ denote this linear mapping, that is $\nabla_{\phi}=\langle\nabla, \phi\rangle$. For higher order differentials we introduce the notation

$$
\nabla_{\phi_{1}} \cdots \nabla_{\phi_{n}}=\left\langle\nabla, \phi_{1}\right\rangle \cdots\left\langle\nabla, \phi_{n}\right\rangle=\left\langle\nabla \otimes \cdots \otimes \nabla, \phi_{n} \otimes \cdots \otimes \phi_{n}\right\rangle .
$$

Define the function $l(x):=\ln (1+x)$, which is analytic in a neighbourhood of zero and satisfies $l(0)=0$. Therefore the function

$$
\widetilde{e}(\phi, \omega):=(e(\cdot, \omega) \circ l)(\phi)=\exp (\langle\omega, \ln (1+\phi)\rangle-\langle 1 \otimes \dot{v}, \phi\rangle),
$$

is analytic as a function of $\phi \in \widetilde{\mathscr{S}}_{q_{0}}(X)$ for functions $\phi \in \widetilde{\mathscr{S}}_{q_{0}}(X)$ satisfying $\phi(x)>-1$, for all $x \in X$. For $\phi_{1}, \ldots, \phi_{n} \in \tilde{\mathscr{S}}(X)$ and $\omega \in \mathscr{\mathscr { S }}^{\prime}(X)$, define

$$
P\left(\phi_{1}, \ldots, \phi_{n} ; \omega\right):=\left.\nabla_{\phi_{1}}^{\theta} \cdots \nabla_{\phi_{n}}^{\theta} \widetilde{e}(\theta, \omega)\right|_{\theta=0} .
$$

From the analyticity at zero of $\widetilde{e}(\theta, \omega)$, we obtain the following expansion

$$
\widetilde{e}(t \theta, \omega)=\sum_{n=0}^{\infty} \frac{t^{n}}{n !} P(\theta, \ldots, \theta ; \omega),
$$

which is convergent in a neighbourhood of zero. Moreover,

$$
\begin{aligned}
P\left(\phi_{1}, \ldots, \phi_{n} ; \omega\right) & =\left\langle\left.\nabla^{\theta} \otimes \cdots \otimes \nabla^{\theta} \widetilde{e}(\theta, \omega)\right|_{\theta=0}, \phi_{1} \otimes \cdots \otimes \phi_{n}\right\rangle \\
& =\left\langle P_{n}(\omega), \phi_{1} \otimes \cdots \otimes \phi_{n}\right\rangle,
\end{aligned}
$$

where

$$
P_{n}(\omega):=\left.\nabla^{\theta} \otimes \cdots \otimes \nabla^{\theta} \widetilde{e}(\theta, \omega)\right|_{\theta=0} .
$$

We see that $\left\langle P_{n}(\omega), \phi^{(n)}\right\rangle$ is well defined for all functions $\phi^{(n)} \in \tilde{\mathscr{S}}(X)^{\hat{\otimes} n}$, where $\widetilde{\mathscr{S}}(X)^{\otimes}$ is the symmetric tensor product of $\widetilde{\mathscr{S}}(X)$ with itself taken $n$ times. We remark that this function space is equal to the space of all functions $f \in \tilde{\mathscr{S}}\left(X^{n}\right)$ that satisfies $f=f\left(x_{1}, \ldots, x_{n}\right)$ is symmetric $\pi^{\times n}$-almost everywhere in the variables $x_{1}, \ldots, x_{n} \in X$ (see, (3.5) for more details). According to [1] and [13],

$$
\left\{\left\langle P_{n}(\omega), \phi^{(n)}\right\rangle: n \in \mathrm{N} \cup\{0\}, \phi^{(n)} \in \tilde{\mathscr{S}}(X)^{\hat{\otimes} n}\right\},
$$

forms a total set in $L^{2}\left(\mu_{\pi}\right)$ and

$$
\widetilde{e}(\phi, \omega)=\sum_{n=0}^{\infty} \frac{1}{n !}\left\langle P_{n}(\omega), \phi^{\otimes n}\right\rangle .
$$


From the form of $\widetilde{e}(\cdot)$ we see that the polynomials $P_{n}$ are generalized Charlier polynomials. In order to emphasise this, we will in the sequel write $C_{n}(\cdot):=$ $P_{n}(\cdot)$.

Proposition 3.1. For any $m, n \in \mathbf{N}, \phi^{(n)} \in \widetilde{\mathscr{S}}(X)^{\hat{\otimes} n}$ and $\psi^{(m)} \in \tilde{\mathscr{S}}(X)^{\hat{\otimes} m}$,

$$
\int_{\widetilde{\mathscr{I}}^{\prime}(X)}\left\langle C_{n}(\omega), \phi^{(n)}\right\rangle\left\langle C_{m}(\omega), \psi^{(m)}\right\rangle d \mu_{\pi}(\omega)=\delta_{n, m} n !\left(\phi^{(n)}, \psi^{(n)}\right)_{\pi} .
$$

Proof. The proof is similar to the proof of [13, Prop. 2.3]. Choose $\phi, \psi \in$ $\tilde{\mathscr{S}}(X)$ and $z_{1}, z_{2} \in \mathrm{C}$. Then

$$
\begin{aligned}
\int_{\widetilde{\mathscr{S}}^{\prime}(X)} & \widetilde{e}\left(z_{1} \phi, \omega\right) \widetilde{e}\left(z_{2} \psi, \omega\right) d \mu_{\pi}(\omega) \\
= & \exp \left(-\left\langle 1 \otimes \dot{v}, z_{1} \phi+z_{2} \psi\right\rangle\right) \\
& \int_{\widetilde{\mathscr{P}}^{\prime}(X)} \exp \left(\left\langle\omega, \ln \left(1+z_{1} \phi\right)+\ln \left(1+z_{2} \psi\right)\right\rangle\right) d \mu_{\pi}(\omega) \\
= & \exp \left(-\left\langle 1 \otimes \dot{v}, z_{1} \phi+z_{2} \psi\right\rangle\right) \\
& \int_{\widetilde{\mathscr{P}}^{\prime}(X)} \exp \left(\left\langle\omega, \ln \left(\left(1+z_{1} \phi\right)\left(1+z_{2} \psi\right)\right)\right\rangle\right) d \mu_{\pi}(\omega) \\
= & \exp \left(z_{1} z_{2}\langle 1 \otimes \dot{v}, \phi \psi\rangle\right) \\
= & \exp \left(z_{1} z_{2}(\phi, \psi)_{\pi}\right) \\
= & \sum_{n=0}^{\infty} \frac{z_{1} z_{2}}{n !}\left(\phi^{\otimes n}, \psi^{\otimes n}\right)_{\pi} .
\end{aligned}
$$

On the other hand, we can use (3.2) to get

$$
\begin{aligned}
\int_{\widetilde{\mathscr{I}}^{\prime}(X)} & \widetilde{e}\left(z_{1} \phi, \omega\right) \widetilde{e}\left(z_{2} \psi, \omega\right) d \mu_{\pi}(\omega) \\
= & \sum_{n, m=0}^{\infty} \frac{z_{1}^{n} z_{2}^{m}}{n ! m !} \int_{\widetilde{\mathscr{S}}^{\prime}(X)}\left\langle C_{n}(\omega), \phi^{\otimes n}\right\rangle\left\langle C_{m}(\omega), \psi^{\otimes m}\right\rangle d \mu_{\pi}(\omega) .
\end{aligned}
$$

By comparing the coefficients in the expressions (3.3) and (3.4), we see that the result holds for $\phi^{(n)}=\left(z_{1} \phi\right)^{\otimes n}$ and $\psi^{(m)}=\left(z_{2} \psi\right)^{\otimes m}$. The proof now follows by linearity and the polarization identity, which enables us to extend the result to $\phi^{(n)} \in \widetilde{\mathscr{S}}(X)^{\hat{\otimes} n}$ and $\psi^{(m)} \in \widetilde{\mathscr{S}}(X)^{\hat{\otimes} m}$. 
Definition 3.2. A function $F: \tilde{\mathscr{S}}^{\prime}(X) \rightarrow \mathrm{C}$ of the form

$$
F(\omega)=\sum_{n=0}^{N}\left\langle\omega^{\otimes n}, \phi^{(n)}\right\rangle, \quad \omega \in \tilde{\mathscr{S}}(X), N \in \mathrm{N},
$$

is called a continuous polynomial if $\phi^{(n)} \in \widetilde{\mathscr{S}}_{\mathrm{C}}(X)^{\hat{\otimes} n}$ (the complexification of $\left.\widetilde{\mathscr{S}}(X)^{\hat{\otimes} n}\right)$. We denote the space of such continuous polynomials by $\mathscr{P}\left(\widetilde{\mathscr{S}}^{\prime}(X)\right)$.

Corollary 3.3 (non-degeneracy of $\mu_{\pi}$ ). For all $F \in \mathscr{P}\left(\tilde{\mathscr{S}}^{\prime}(X)\right.$ ) for which $F=0, \mu_{\pi}$-almost everywhere, we have $F(\omega)=0$ for all $\omega \in \widetilde{\mathscr{S}}^{\prime}(X)$.

Proof. It can be shown that each continuous polynomial $F$ admits a representation of the form

$$
F(\omega)=\sum_{n=0}^{N}\left\langle C_{n}(\omega), \hat{\phi}_{n}\right\rangle,
$$

where $\hat{\phi}_{n} \in \tilde{\mathscr{S}}(X)^{\hat{\otimes} n}$. Without loss of generality, take $F(\omega)=\left\langle C_{n}(\omega), \hat{\phi}_{n}\right\rangle$. By Proposition 3.1,

$$
0=\mathrm{E}_{\mu_{\pi}}\left[\left\langle C_{n}(\omega), \hat{\phi}_{n}\right\rangle^{2}\right]=\int_{X} \phi_{n}^{2}(x) \pi(d x) .
$$

Hence, $\phi_{n} \in \mathcal{N}_{\pi}$, which implies that $\hat{\phi}_{n}$ is the null element in $\tilde{\mathscr{S}}(X)$. Therefore $F$ is identically zero.

Remark 3.4. A probability measure with the property in Corollary 3.3 is called non-degenerate (see [12]). We will make use of this property for the construction of stochastic test function and distribution spaces.

For functions $f: X^{n} \rightarrow \mathrm{R}$ define the symmetrization $\tilde{f}$ of $f$ by

$$
\widetilde{f}\left(x_{1}, \ldots, x_{n}\right):=\frac{1}{n !} \sum_{\sigma} f\left(x_{\sigma_{1}}, \ldots, x_{\sigma_{n}}\right),
$$

for all permutations $\sigma$ of $\{1, \ldots, n\}$. We call a function $f: X^{n} \rightarrow \mathrm{R}$ symmetric if $\widetilde{f}=f$, that is a function is symmetric if it is equal to its symmetrization. Denote by $L_{s}^{2}\left(X^{n}, \pi^{\times n}\right)$ the space of all symmetric functions on $X^{n}$ which are square integrable with respect to $\pi^{\times n}$. Let $f_{n}$ be a function in $L_{s}^{2}\left(X^{n}, \pi^{\times n}\right)$. Since $\mathscr{S}(X)$ is dense in $L^{2}(\pi)$, we can find a sequence of functions $f_{n}^{(m)} \in$ $\tilde{\mathscr{S}}(X)^{\hat{\otimes} n}$ such that $f_{n}^{(m)}$ converges to $f_{n}$ in $L_{s}^{2}\left(X^{n}, \pi^{\times n}\right)$ as $m \rightarrow \infty$. Define $\left\langle C_{n}(\omega), f_{n}\right\rangle$ by

$$
\left.\left\langle C_{n}(\omega), f_{n}\right\rangle=\lim _{m \rightarrow \infty}\left\langle C_{n}(\omega), f_{n}^{(m)}\right\rangle, \quad \text { (limit in } L^{2}\left(\mu_{\pi}\right)\right) .
$$


We remark that this limit exists by Proposition 3.1. Moreover, let $f_{n}^{(j)} \in$ $\widetilde{\mathscr{S}}(X)^{\hat{\otimes} n}$ be another sequence converging to $f_{n}$. Then by Proposition 3.1,

$$
\int_{\tilde{\mathscr{S}}^{\prime}(X)}\left(\left\langle C_{n}(\omega), f_{n}^{(m)}\right\rangle-\left\langle C_{n}(\omega), f_{n}^{(j)}\right\rangle\right)^{2} d \mu_{\pi}(\omega)=n !\left|f_{n}^{(m)}-f_{n}^{(j)}\right|_{\pi}^{2},
$$

which shows that the definition of $\left\langle C_{n}(\omega), f_{n}\right\rangle$ is independent of the choice of approximating sequence $\left\{f_{n}^{(m)}\right\}_{m=1}^{\infty}$. From the calculation,

$$
\begin{aligned}
\left.\nabla_{\phi} \widetilde{e}(\eta, \omega)\right|_{\eta=0} & =\left.\frac{d}{d \epsilon} \exp (\langle\omega, \ln (1+\eta+\epsilon \phi)\rangle-\langle 1 \otimes \dot{v}, \eta+\epsilon \phi\rangle)\right|_{\epsilon=0, \eta=0} \\
& =\langle\omega-1 \otimes \dot{v}, \phi\rangle,
\end{aligned}
$$

it follows that the first Charlier polynomial $C_{1}$ is given by $C_{1}(\omega)=\omega-1 \otimes \dot{v}$. From Proposition 3.1, we therefore obtain the following well known isometry.

Lemma 3.5. Let $f \in L^{2}(X, \pi)$. Then

$$
\int_{\tilde{\mathscr{S}}^{\prime}(X)}\langle\omega-1 \otimes \dot{v}, f\rangle^{2} d \mu_{\pi}(\omega)=|f|_{\pi}^{2}
$$

For any Borel sets $\Lambda_{1} \subset \mathrm{R}^{d}$ and $\Lambda_{2} \subset \mathrm{R}_{0}$ such that 0 is not in the closure of $\Lambda_{2}$, we define the random measures

$$
N\left(\Lambda_{1}, \Lambda_{2}\right):=\left\langle\omega, 1_{\Lambda_{1} \times \Lambda_{2}}\right\rangle \quad \text { and } \quad \tilde{N}\left(\Lambda_{1}, \Lambda_{2}\right):=\left\langle\omega-1 \otimes \dot{v}, 1_{\Lambda_{1} \times \Lambda_{2}}\right\rangle .
$$

From the characteristic function of $\mu_{\pi}$ it is clear that $N$ is a Poisson random measure, and $\widetilde{N}$ the corresponding compensated Poisson random measure. Moreover, the compensator of $N\left(\Lambda_{1}, \Lambda_{2}\right)$ is given by $\left\langle 1 \otimes \dot{v}, 1_{\Lambda_{1} \times \Lambda_{2}}\right\rangle$, which is equal to $\pi\left(\Lambda_{1}, \Lambda_{2}\right)$. We have therefore justified the following identity

$$
\int_{X} \phi(x) \tilde{N}(d x)=\langle\omega-1 \otimes \dot{v}, \phi\rangle, \quad \phi \in L^{2}(X, \pi) .
$$

So, in a generalized sense, the compensated Poisson random measure $\tilde{N}$ has a Radon-Nikodym derivative with respect to the Lebesgue measure on $X$, which is given by $\omega-1 \otimes \dot{v}$. Every square integrable pure jump Lévy process $L$ has a representation

$$
L_{t}=\alpha t+\int_{0}^{t} \int_{\mathrm{R}_{0}} z \tilde{N}(d t, d z)
$$

From (3.7) we see that such Lévy processes $L$ admits the representation

$$
L_{t}=\alpha t+\left\langle\omega-1 \otimes \dot{v}, f_{t}\right\rangle, \quad f_{t}(x, z)=z 1_{x \leq t} .
$$


As a stochastic distribution, this representation is valid also when $L$ is not square integrable.

\section{Stochastic test and distribution functions}

In this section we will define spaces of test functions and distributions as pairs of dual spaces with respect to the inner product $(\cdot, \cdot)_{\pi}$, and stochastic test functions and stochastic distributions as pairs of dual spaces with respect to the inner product on $L^{2}\left(\mu_{\pi}\right)$. Our approach is a variation of the approach taken in [11], and our system of generalized Charlier polynomials coincides with the generalized Appell systems in [12], which provides in the same manner stochastic test function and distribution spaces (compare, [12, Example 27]).

Observe that for $\phi \in \widetilde{\mathscr{S}}(X)$, we have by Theorem 2.3, that (4.1)

$(\phi, \phi)_{\pi}=\left\langle 1 \otimes \dot{v}, \phi^{2}\right\rangle \leq\|1 \otimes \dot{v}\|_{-q_{0}, \pi}\left\|\phi^{2}\right\|_{q_{0}, \pi} \leq M_{q_{0}}\|1 \otimes \dot{v}\|_{-q_{0}, \pi}\|\phi\|_{q_{0}, \pi}^{2}$,

from which it follows that $\tilde{\mathscr{S}}(X)$ is contained in $L^{2}(X, \pi)$. Define $\tilde{\mathscr{S}}^{\prime}(X)$ as the dual of $\widetilde{\mathscr{S}}(X)$. It follows that the topology on $\widetilde{\mathscr{S}}^{\prime}(X)$ is induced by the following compatible system of norms

$$
|\phi|_{-p, \pi}:=\|(1 \otimes \dot{v}) \phi\|_{-p, \pi}, \quad \text { for } \quad p \geq p_{0} .
$$

We will now construct the Kondratiev type stochastic test function space and the Kondratiev stochastic distribution space. Let $f \in \mathscr{P}\left(\widetilde{\mathscr{S}}^{\prime}(X)\right)$. By Corollary $3.3, f$ has a unique representation

$$
f(\omega)=\sum_{n=0}^{N}\left\langle C_{n}(\omega), f_{n}\right\rangle, \quad f_{n} \in \tilde{\mathscr{S}}(X)^{\hat{\otimes} n} .
$$

For any natural number $p \geq q_{0}$, define the Hilbert space $(\mathscr{S})_{p}^{1}$ as the completion of $\mathscr{P}:=\mathscr{P}\left(\tilde{\mathscr{S}}^{\prime}(X)\right)$ with respect to the norm

$$
\|f\|_{p, 1, K}^{2}=\sum_{n=0}^{\infty}(n !)^{2}\left\|f_{n}\right\|_{p, \pi}^{2}, \quad f \in \mathscr{P} .
$$

The corresponding inner product is

$$
((f, g))_{p, 1, K}=\sum_{n=0}^{\infty}(n !)^{2}\left(f_{n}, g_{n}\right)_{p},
$$

where $(\cdot, \cdot)_{p}$ denotes the inner product on $\tilde{\mathscr{S}}_{p}(X)^{\hat{\otimes} n}$. Obviously, $(\mathscr{S})_{p+1}^{1} \subset$ $(\mathscr{S})_{p}^{1}$. We define the space $(\mathscr{S})^{1}$ as the projective limit of $(\mathscr{S})_{p}^{1}$. By [1, Thm. 4], 
$(\mathscr{S})^{1}$ is a nuclear Fréchet space which is densely topologically embedded in $L^{2}\left(\mu_{\pi}\right)$.

For every natural number $p$ define the space $(\mathscr{S})_{-p}^{-1}$ as the dual of $(\mathscr{S})_{p}^{1}$. We have the inclusion $(\mathscr{S})_{-p}^{-1} \subset(\mathscr{S})_{-(p+1)}^{-1}$. Denote by $(\mathscr{S})^{-1}$ the inductive limit of $(\mathscr{S})_{-p}^{-1}$, which is equal to the dual of $(\mathscr{S})^{1}$. Let $\langle\langle\cdot, \cdot\rangle\rangle$ denote the dual pairing between $(\mathscr{S})^{1}$ and $(\mathscr{S})^{-1}$. Moreover, for any $F(\omega)=\sum_{n=0}^{\infty}\left\langle C_{n}(\omega), F_{n}\right\rangle$ with kernels $F_{n} \in \tilde{\mathscr{S}}^{\prime}(X)^{\otimes}$, define the norm

$$
\|F\|_{-p,-1, K}^{2}:=\sum_{n=0}^{\infty}\left|F_{n}\right|_{-p, \pi}^{2} .
$$

We equip $(\mathscr{S})^{-1}$ with the inductive limit topology induced by the norms $\|F\|_{-p,-1, K}^{2}$. The next results provide a characterization of $F \in(\mathscr{S})^{-1}$ and the dual pairing $\langle\langle\cdot, \cdot\rangle\rangle$. The proof is standard, so it is omitted.

Lemma 4.1. $F \in(\mathscr{S})^{-1}$ if and only if $F$ admits an expansion $F(\omega)=$ $\sum_{n=0}^{\infty}\left\langle C_{n}(\omega), F_{n}\right\rangle$, where $F_{n} \in \widetilde{\mathscr{S}}^{\prime}(X)^{\hat{\otimes} n}$ and $\|F\|_{-p,-1, K}<\infty$, for some $p>q_{0}$. If $f(\omega)=\sum_{n=0}^{\infty}\left\langle C_{n}(\omega), f_{n}\right\rangle \in(\mathscr{S})^{1}$, then

$$
\langle\langle F, f\rangle\rangle=\sum_{n=0}^{\infty} n !\left(F_{n}, f_{n}\right)_{\pi} .
$$

Moreover, $\langle\langle\cdot, \cdot\rangle\rangle$ is an extension of the inner product on $L^{2}\left(\mu_{\pi}\right)$.

\section{The $\mathscr{S}$-transform and Wick products}

There are several transformations from spaces of stochastic distributions to spaces of deterministic functionals. In this paper we will focus on the $\mathscr{S}$ transform. Our approach is based on the results in [1], [12], [13] and [21].

Let $F \in(\mathscr{S})^{-1}$. Then there exists a natural number $p(F)>0$ such that $F \in(\mathscr{S})_{-p(F)}^{-1}$. Denote by $U_{p}$ the set

$$
U_{p}:=\left\{\phi \in \tilde{\mathscr{S}}(X):\|\phi\|_{p, \pi}<1\right\} .
$$

For any $\phi \in \tilde{\mathscr{S}}(X)$, it follows from the chaos expansion of $\widetilde{e}(\phi)$ given by equation (3.2) that

$$
\|\widetilde{e}(\phi, \omega)\|_{p, 1, K}^{2}=\sum_{n=0}^{\infty}\|\phi\|_{p, \pi}^{2 n} .
$$

Hence, $\widetilde{e}(\phi) \in(\mathscr{S})_{p}^{1}$ if and only if $\phi \in U_{p}$. 
Definition 5.1. Let $F \in(\mathscr{S})_{-p}^{-1}$ and $\xi \in U_{p}$. We define the $\mathscr{S}$-transform of $F$ by

$$
\mathscr{S}(F)(\xi):=\langle\langle F(\omega), \widetilde{e}(\xi, \omega)\rangle .
$$

Observe that since $F \in(\mathscr{S})_{-p}^{-1}$ and $\widetilde{e}(\xi) \in(\mathscr{S})_{p}^{1}$, it follows that $|\mathscr{S}(F)(\xi)|<$ $\infty$.

Lemma 5.2. Let $F=\sum_{n=0}^{\infty}\left\langle C_{n}(\omega), F_{n}\right\rangle \in(\mathscr{S})_{-p}^{-1}$. Then

$$
\mathscr{S}(F)(\xi)=\sum_{n=0}^{\infty}\left(F_{n}, \xi^{\otimes n}\right)_{\pi}, \quad \xi \in U_{p} .
$$

Proof. Let $\xi \in U_{p}$. By Proposition 3.1 and the chaos expansion of $\widetilde{e}(\xi)$ given by (3.2),

$$
\mathscr{S}(F)(\xi)=\left\langle\left\langle\sum_{n=0}^{\infty}\left\langle C_{n}(\omega), F_{n}\right\rangle, \sum_{m=0}^{\infty}\left\langle C_{m}(\omega), \frac{1}{m !} \xi^{\otimes m}\right\rangle\right\rangle\right\rangle=\sum_{n=0}^{\infty}\left(F_{n}, \xi^{\otimes n}\right)_{\pi} .
$$

Denote by $l^{-1}$ the inverse of the function $l(x)=\ln (1+x)$, that is $l^{-1}(x)=$ $e^{x}-1$. Define another transform denoted $\mathscr{S}_{P}(F)$ by:

$$
\mathscr{S}_{P}(F)(\xi):=\langle\langle F(\omega), e(\xi, \omega)\rangle\rangle
$$

where

$$
e(\phi, \omega):=\exp \left(\langle\omega, \phi\rangle-\left\langle 1 \otimes \dot{v}, e^{\phi}-1\right\rangle\right) .
$$

For every $\xi$ such that $e^{\xi}-1 \in U_{p}$ and $F=\sum_{n=0}^{\infty}\left\langle C_{n}(\omega), F_{n}\right\rangle \in(\mathscr{S})_{-p}^{-1}$, we have that

$$
\mathscr{S}_{P}(F)(\xi)=\sum_{n=0}^{\infty}\left(F_{n},\left(e^{\xi}-1\right)^{\otimes n}\right)_{\pi}=\left(\mathscr{S}(F) \circ l^{-1}\right)(\xi) .
$$

Denote by $\mathscr{U}=\operatorname{Hol}\left(\theta_{0}\right)$, the algebra of germs of functions that are holomorphic in a neighbourhood of $\theta_{0}$ (See, [1], [4] and [16] for more details). We equip $\mathscr{U}$ with the inductive limit topology induced by the norms

$$
|g|_{p, l, \infty}:=\sup _{\|\phi\|_{p, \pi} \leq 2^{-l}}|g(\phi)|, \quad p, l \in \mathbf{N},
$$

(see, [4]). A characterization of the image of $(\mathscr{S})^{-1}$ under the $\mathscr{S}_{P}$-transform was proved in [1]. Note that the function $l^{-1}$ is analytic in a neighbourhood of 
zero. By equation (5.2) and the analyticity of $l^{-1}$, we deduce that the characterization of the image of the $\mathscr{S}_{P}$-transform in [1] also is valid for the $\mathscr{S}$-transform. Therefore, by [1, Thm. 5],

Theorem 5.3. If $F \in(\mathscr{S})^{-1}$ then $\mathscr{S}(F) \in \operatorname{Hol}(0)$. Conversely, if $G \in$ $\operatorname{Hol}(0)$ there is a uniquely defined distribution $F \in(\mathscr{S})^{-1}$ such that $\mathscr{S}(F)=$ $G$ on some neighbourhood of zero in $(\mathscr{S})_{\mathrm{C}}^{1}$ (the complexification of $(\mathscr{S})^{1}$ ).

By Theorem 5.3, the $\mathscr{S}$-transform is an isomorphism between $(\mathscr{S})^{-1}$ and $\mathscr{U}$. Moreover, if $f, g \in \operatorname{Hol}(0)$ then $f g \in \operatorname{Hol}(0)$. Hence the following definition is well defined,

Definition 5.4 (Wick product). Let $F, G \in(\mathscr{S})^{-1}$. Define the wick product, denoted by $F \diamond G$, of $F$ and $G$, by

$$
F \diamond G:=\mathscr{S}^{-1}(\mathscr{S}(F) \mathscr{S}(G)) .
$$

It follows directly from the properties of the $\mathscr{S}$-transform and the definition of the Wick product that Wick multiplication is a continuous operation. Moreover, we have the following characterization of the Wick product in terms of chaos expansions.

Proposition 5.5. Let $F(\omega)=\sum_{n=0}^{\infty}\left\langle C_{n}(\omega), F_{n}\right\rangle$ and let $G(\omega)=$ $\sum_{n=0}^{\infty}\left\langle C_{n}(\omega), G_{n}\right\rangle$ be elements in $(\mathscr{S})^{-1}$. Then $F \diamond G \in(\mathscr{S})^{-1}$ and

$$
(F \diamond G)(\omega)=\sum_{k=0}^{\infty}\left(\sum_{n+m=k}\left\langle C_{k}(\omega), F_{n} \hat{\otimes} G_{m}\right\rangle\right) .
$$

Proof. The proof is similar to proof for the Brownian motion case provided in [8], so it is omitted.

For $F \in(\mathscr{S})^{-1}$, define $F^{\diamond n}:=F \diamond \cdots \diamond F$ (the Wick product taken $n$ times). Moreover, define the Wick exponential of $F$, denoted $\exp ^{\diamond}(F)$, by

$$
\exp ^{\diamond}(F):=\sum_{n=0}^{\infty} \frac{1}{n !} F^{\diamond n},
$$

whenever $\sum_{n=0}^{\infty} \frac{1}{n !} F^{\diamond n} \in(\mathscr{S})^{-1}$. If $\phi \in \tilde{\mathscr{S}}^{\prime}(X)$, it follows from Lemma 4.3 that $\left\langle C_{1}(\omega), \phi\right\rangle \in(\mathscr{S})^{-1}$. By Proposition 5.5,

$$
\left\langle C_{1}(\omega), \phi\right\rangle^{\diamond n}=\left\langle C_{n}(\omega), \phi^{\otimes n}\right\rangle .
$$

From the chaos expansion of $\widetilde{e}(\phi, \omega)$ given by (3.2), we therefore have that

$$
\widetilde{e}(\phi, \omega)=\exp ^{\diamond}(\langle\omega-1 \otimes \dot{v}, \phi\rangle),
$$


for all $\phi \in \tilde{\mathscr{S}}^{\prime}(X)$ that satisfies $\phi(x)>-1$, for all $x \in X$.

\section{A unitary isomorphism and configuration spaces}

In this section we will establish a unitary isomorphism between $L^{2}\left(\mu_{\pi}\right)$ and the classical Poisson space with intensity $\pi=\pi_{v}$ defined via the configuration space. This isomorphism can be used to transfer analytical structures from the Poisson space to $L^{2}\left(\mu_{\pi}\right)$.

We need the definition of a configuration space. As in [13] and [11], we introduce the configuration space $\Gamma$ over $X=\mathrm{R}^{d} \times \mathrm{R}_{0}$ by

$$
\Gamma:=\{\gamma \subset X: \operatorname{card}(\gamma \cap K)<\infty \text { for any compact } K \subset X\} .
$$

Denote by $\epsilon_{x}$ the Dirac measure at the point $x \in X$. The correspondence

$$
\Gamma \ni \gamma \longmapsto d \gamma:=\sum_{x \in \gamma} \epsilon_{x} \in \mathscr{M}_{+}(X)
$$

provides a one-to-one mapping $\Phi$ from $\Gamma$ into the space of positive integer valued measures $\mathscr{M}_{+}(X)$ over $\mathscr{B}(X)$. We endow $\Gamma$, as a closed subset of $\mathscr{M}_{+}(X)$, with the relative vague topology. That is, a sequence of measures $\sigma_{n}$ converges to $\sigma$ in $\Gamma$, if and only if for every $f \in C_{c}(X)$, (i.e. the space of continuous functions with compact support) we have

$$
\int_{X} f(x) d \sigma_{n}(x) \longrightarrow \int_{X} f(x) d \sigma(x), \quad \text { as } \quad n \longrightarrow \infty .
$$

Then the continuous functionals

(6.2) $\Gamma \ni \gamma \longmapsto\langle\gamma, f\rangle:=\int_{X} f(x) d \gamma(x)=\sum_{x \in \gamma} f(x), \quad f \in C_{c}(X)$,

induce the topology of $\Gamma$. We need the following key observation regarding the support of $\mu_{\pi}$.

Proposition 6.1.

$$
\mu_{\pi}\left(\left\{\sum_{x \in \gamma} \epsilon_{x} \in \tilde{\mathscr{S}}^{\prime}(X): \gamma \in \Gamma\right\}\right)=1
$$

where the Dirac measure $\epsilon_{x}$ is naturally identified with the corresponding delta function in $\widetilde{\mathscr{S}}^{\prime}(X)$.

Proof. Relation (6.3) follows from the path properties of Lévy processes (see, e.g. [15]). More specifically, almost all the paths of a pure jump Lévy 
process are right-continuous step functions with existing left limits (càdlàg), whose jumps are contained in the support of the Lévy measure (see [13]).

Furthermore, we define the Poisson measure $\mu_{\pi}^{\Gamma}$, with intensity measure $\pi$, on the Borel $\sigma$-algebra $\mathscr{B}(\Gamma)$ as follows.

Definition 6.2. The Laplace transform of $\mu_{\pi}^{\Gamma}$ is given by

$$
l_{\mu_{\pi}^{\Gamma}}(\phi)=\int_{\Gamma} \exp (\langle\gamma, \phi\rangle) d \mu_{\pi}^{\Gamma}(\gamma)=\exp \left(\int_{X}\left(e^{\phi(x)}-1\right) d \pi(x)\right),
$$

where $\phi \in \tilde{\mathscr{S}}(X)$. The existence of $\mu_{\pi}^{\Gamma}$ follows from Proposition 6.1 and the identification in relation (6.1).

Taking into account that we have

$$
\int_{\tilde{\mathscr{S}}^{\prime}(X)} e^{\langle\omega, \phi\rangle} d \mu_{\pi}(\omega)=: l_{\mu_{\pi}}(\phi)=l_{\mu_{\pi}^{\Gamma}}(\phi),
$$

for all $\phi \in \tilde{\mathscr{S}}(X)$, we conclude that the measure $\mu_{\pi}$ is the image of $\mu_{\pi}^{\Gamma}$ under the mapping $\Phi: \Gamma \rightarrow \Phi(\Gamma)=: \Omega$ in (6.1). That is for all $B \in \mathscr{B}\left(\widetilde{\mathscr{S}}^{\prime}(X)\right)$,

$$
\mu_{\pi}(B)=\mu_{\pi}(B \cap \Omega)=\mu_{\pi}^{\Gamma}\left(\Phi^{-1}(B \cap \Omega)\right) .
$$

From relation (6.5), together with the change of variable formula for the Lebesgue integral, it follows that for all $f \in L^{1}\left(\Gamma, \mu_{\pi}^{\Gamma}\right)$, the function $f \circ \Phi^{-1}$ is in $L^{1}\left(\Omega, \mu_{\pi}\right)$ and

$$
\int_{\Gamma} f(\gamma) d \mu_{\pi}^{\Gamma}(\gamma)=\int_{\Omega} f \circ \Phi^{-1}(\omega) d \mu_{\pi}(\omega) .
$$

Thus, we have proved the following result.

TheOREM 6.3. The map $U_{\Phi}: L^{2}\left(\mu_{\pi}\right) \rightarrow L^{2}\left(\mu_{\pi}^{\Gamma}\right)$ given by

$$
g \longmapsto g \circ \Phi
$$

is a unitary isomorphism.

REMARK 6.4. It is important to note that the measure $\mu_{\pi} \underset{\sim}{\text { does not ad- }}$ mit the construction of a satisfactory differential calculus on $\tilde{\mathscr{S}}^{\prime}(X)$. Since $\mu_{\pi}(\xi+\phi) \perp \mu_{\pi}(\xi)$ (see, e.g., [6]), integration by parts and adjoint operators are not available. We overcome this circumstance by using the unitary isomorphism of Theorem 6.3 to transport analytical structures from $L^{2}\left(\mu_{\pi}^{\Gamma}\right)$ to $L^{2}\left(\mu_{\pi}\right)$ (compare, [13]). We will make use of this principle in Section 7, where we introduce the Poissonian gradient for Lévy processes. 


\section{The Poissonian gradient and the Skorohod integral}

In this section we explore some properties of the Poissonian gradient and the Skorohod integral on the Poisson space. Most of the results in this regard are extensions of results obtained in [17]. We proceed to show how the Skorohod integral behaves under the $\mathscr{S}$-transform.

Define a space $\mathrm{D} \subset L^{2}\left(\mu_{\pi}\right)$ by

$$
\mathrm{D}:=\left\{f(\omega)=\sum_{n=0}^{\infty}\left\langle C_{n}(\omega), f_{n}\right\rangle: \sum_{n=0}^{\infty} n n !\left|f_{n}\right|_{\pi}^{2}<\infty\right\},
$$

and a linear operator $D: \mathrm{D} \rightarrow L^{2}\left(\pi \times \mu_{\pi}\right)$ by

$$
D_{x} f(\omega):=\sum_{n=1}^{\infty}\left\langle C_{n-1}(\omega), n f_{n}(\cdot, x)\right\rangle,
$$

for $f(\omega)=\sum_{n=0}^{\infty}\left\langle C_{n}(\omega), f_{n}\right\rangle \in \mathrm{D}$, where $f_{n}(\cdot, x)$ is the function $f_{n}$ with the last argument $x=\left(x_{1}, \ldots, x_{d+1}\right) \in X$ held fixed. It can be seen from a direct calculation that

$$
\|D f\|_{L^{2}\left(\pi \times \mu_{\pi}\right)}^{2}=\sum_{n=1}^{\infty} n n !\left|f_{n}\right|_{\pi}^{2} .
$$

Thus, $D f \in L^{2}\left(\pi \times \mu_{\pi}\right)$ whenever $f \in \mathrm{D}$. Note that $(\mathscr{S})^{1} \subseteq \mathrm{D}$. Define another linear operator, called the Poissonian gradient, denoted by $\nabla^{P}$,

$$
\left(\nabla^{P} f\right)(\gamma, x)=f\left(\gamma+\epsilon_{x}\right)-f(\gamma), \quad \gamma \in \Gamma, x \in X,
$$

for all variables of the form $f(\gamma)=g\left(\left\langle\gamma_{\tilde{A}} \phi_{1}\right\rangle, \ldots,\left\langle\gamma, \phi_{N}\right\rangle\right)$, where the function $g \in C_{b}^{\infty}\left(\mathrm{R}^{N}\right)$ and $\phi_{1}, \ldots, \phi_{N} \in \tilde{\mathscr{S}}(X)$. Notice that $\nabla^{P}$ is defined on $L^{2}\left(\mu_{\pi}^{\Gamma}\right)$. We define the operator $\widetilde{\nabla}^{P}$ on $L^{2}\left(\mu_{\pi}\right)$ by

$$
\widetilde{\nabla}^{P}=U_{\Phi}^{-1} \nabla^{P} U_{\Phi}
$$

Lemma 7.1. The operators $D$ and $\widetilde{\nabla}^{P}$ coincide on a dense subset of $L^{2}\left(\mu_{\pi}\right)$, hence the closure of $D$ equals the closure of $\widetilde{\nabla}^{P}$.

Proof. From the The chaos expansion of $\widetilde{e}(\phi, \omega)$ given by (3.2) and the definition of $D$,

$$
\begin{aligned}
D_{x} \widetilde{e}(\phi, \omega) & =D_{x}\left(\sum_{n=0}^{\infty}\left\langle C_{n}(\omega), \frac{\phi^{\otimes n}}{n !}\right\rangle\right) \\
& =\sum_{n=1}^{\infty}\left\langle C_{n-1}(\omega), \frac{n \phi^{\otimes n-1} \phi(x)}{n !}\right\rangle
\end{aligned}
$$




$$
\begin{aligned}
& =\phi(x) \sum_{n=0}^{\infty}\left\langle C_{n}(\omega), \frac{\phi^{\otimes n}}{n !}\right\rangle \\
& =\phi(x) \widetilde{e}(\phi, \omega) .
\end{aligned}
$$

On the other hand, $\widetilde{\nabla}^{P}$ applied to $\widetilde{e}(\phi, \omega)$ gives

$$
\begin{aligned}
\left(\widetilde{\nabla}^{P} \widetilde{e}(\phi, \omega)\right)(x) & =\left(U_{\Phi}^{-1} \nabla^{P} U_{\Phi} \widetilde{e}(\phi, \omega)\right)(x) \\
& =U_{\Phi}^{-1}\left(\widetilde{e}\left(\phi, \gamma+\epsilon_{x}\right)-\widetilde{e}(\phi, \gamma)\right) \\
& =U_{\Phi}^{-1}\left(\widetilde{e}(\phi, \gamma)\left(e^{\left\lfloor\epsilon_{x}, \ln (1+\phi)\right\rangle}-1\right)\right) \\
& =U_{\Phi}^{-1}(\widetilde{e}(\phi, \gamma) \phi(x)) \\
& =\widetilde{e}(\phi, \omega) \phi(x) .
\end{aligned}
$$

By comparing (7.2) and (7.3), we see that $D_{x} \widetilde{e}(\phi, \omega)=\left(\widetilde{\nabla}^{P} \widetilde{e}(\phi, \omega)\right)(x)$, for all $x \in X$ and $\phi \in \widetilde{\mathscr{S}}(X)$. Since both operators are closable and the linear span of variables of the form $\widetilde{e}(\phi)$ is dense in $L^{2}\left(\mu_{\pi}\right)$ the result follows.

We will now consider generalized random fields $F: X \rightarrow(\mathscr{S})^{-1}$. Observe that the chaos expansion of such fields may be written as

$$
F(x)=\sum_{n=0}^{\infty}\left\langle C_{n}(\omega), F_{n}(\cdot, x)\right\rangle,
$$

where $F_{n}(\cdot, x) \in \tilde{\mathscr{S}}^{\prime}(X)^{\hat{\otimes} n}$ for every $x=\left(x_{1}, \ldots, x_{d+1}\right) \in X$ and $\|F(x)\|_{-p,-1, K}<\infty$, for some natural number $p>0$. Let $\mathrm{L}$ be the set of all $F: X \rightarrow(\mathscr{S})^{-1}$ such that $\widetilde{F}_{n} \in \widetilde{\mathscr{S}}^{\prime}(X)^{\hat{\otimes}(n+1)}$ and

$$
\sum_{n=0}^{\infty}\left|\widetilde{F}_{n}\right|_{-p, \pi}^{2}<\infty,
$$

for some natural number $p>0$.

Definition 7.2. For $F \in \mathrm{L}$, define the Skorohod integral, denoted $\delta(\cdot)$, by

$$
\delta(F):=\sum_{n=0}^{\infty}\left\langle C_{n+1}(\omega), \widetilde{F}_{n}\right\rangle,
$$

where $\widetilde{F}_{n}$ denotes the symmetrization of $F_{n}$.

From the definition of the Skorohod integral conjoint with the assumption on elements of $\mathrm{L}$, it follows that $\delta(F) \in(\mathscr{S})^{-1}$. 
In [2], it was proved that for predictable integrands the Skorohod integral coincides with the usual Itô-type integral with respect to the compensated Poisson random measure $\widetilde{N}$.

The next result shows that the operator $D$, which by Lemma 7.1 is equal to $\widetilde{\nabla}^{P}$, is the dual of the Skorohod integral $\delta$.

Theorem 7.3. Let $F \in \mathrm{L}$ and $f \in(\mathscr{S})^{1}$. Then

$$
\int_{X}\left\langle\left\langle F(x), D_{x} f\right\rangle\right\rangle \pi(d x)=\langle\langle\delta(F), f\rangle\rangle .
$$

Proof. Set $F(x)=\sum_{n=0}^{\infty}\left\langle C_{n}(\omega), F_{n}(\cdot, x)\right\rangle$ and $f=\sum_{m=0}^{\infty}\left\langle C_{m}(\omega), f_{m}\right\rangle$, where $F \in \mathrm{L}$ and $f \in(\mathscr{S})^{1}$. Then $\delta(F) \in(\mathscr{S})^{-1}$. Choose a natural number $p>0$ such that $\delta(F) \in(\mathscr{S})_{-p}^{-1}$. By the Cauchy-Schwartz inequality,

$$
\begin{aligned}
\int_{X}\left|\left(F_{n}(\cdot, x), f_{n+1}(\cdot, x)\right)_{\pi}\right| \pi(d x) & \leq \int_{X^{n+1}}\left|F_{n} f_{n+1}\right| d \pi^{\times(n+1)} \\
& =\int_{X^{n+1}}\left|F_{n}(1 \otimes \dot{v})^{\otimes(n+1)} f_{n+1}\right| d \lambda^{\times(n+1)} \\
& \leq\left\|F_{n}(1 \otimes \dot{v})^{\otimes(n+1)}\right\|_{-p, \pi}\left\|f_{n+1}\right\|_{p, \pi} \\
& =\left|F_{n}\right|_{-p, \pi}\left\|f_{n+1}\right\|_{p, \pi} .
\end{aligned}
$$

By first changing the order of integration and summation using [5, Thm. 2.15], then inequality (7.5) and finally the Cauchy-Schwartz inequality, we get

$$
\begin{aligned}
& \int_{X} \sum_{n=0}^{\infty}(n+1) !\left|\left(F_{n}(\cdot, x), f_{n+1}(\cdot, x)\right)_{\pi}\right| \pi(d x) \\
& \quad=\sum_{n=0}^{\infty}(n+1) ! \int_{X}\left|\left(F_{n}(\cdot, x), f_{n+1}(\cdot, x)\right)_{\pi}\right| \pi(d x) \\
& \quad \leq \sum_{n=0}^{\infty}\left|F_{n}\right|_{-p, \pi}(n+1) !\left\|f_{n}\right\|_{p, \pi} \\
& \quad \leq\left(\sum_{n=0}^{\infty}\left|F_{n}\right|_{-p, \pi}^{2}\right)^{1 / 2}\left(\sum_{n=0}^{\infty}((n+1) !)^{2}\left\|f_{n}\right\|_{p, \pi}^{2}\right)^{1 / 2} \\
& =\|\delta(F)\|_{-p,-1, K}\|f\|_{p, 1, K}<\infty .
\end{aligned}
$$


By the dominated convergence theorem,

$$
\begin{aligned}
\int_{X}\left\langle\left\langle F(x), D_{x} f\right\rangle\right\rangle \pi(d x) & =\int_{X} \sum_{n=0}^{\infty}(n+1) !\left(F_{n}(\cdot, x), f_{n+1}(\cdot, x)\right)_{\pi} \pi(d x) \\
& =\sum_{n=0}^{\infty}(n+1) !\left(F_{n}, f_{n+1}\right)_{\pi} \\
& \stackrel{(*)}{=} \sum_{n=0}^{\infty}(n+1) !\left(\widetilde{F}_{n}, f_{n+1}\right)_{\pi} \\
& =\langle\langle\delta(F), f\rangle,
\end{aligned}
$$

where $(*)$ is due to the fact that $f_{n+1}$ is symmetric, and hence $\left(F_{n}, f_{n+1}\right)_{\pi}$ is equal to $\left(\widetilde{F}_{n}, f_{n+1}\right)_{\pi}$.

We remark that if $\delta(F) \in L^{2}\left(\mu_{\pi}\right)$ and $f \in \mathrm{D} \subset L^{2}\left(\mu_{\pi}\right)$, then equation (7.4) reads

$$
\mathrm{E}\left[\int_{X}\left(F(x) D_{x} f\right) \pi(d x)\right]=\mathrm{E}[\delta(F) f] .
$$

According to Theorem 7.3, $\delta=\left(\widetilde{\nabla}^{P}\right)^{*}$. By using the Mecke identity, one can show that (see, e.g., [13])

$$
\left(\left(\nabla^{P}\right)^{*} F\right)(\gamma)=\int_{X} F\left(\gamma-\epsilon_{x}, x\right) d \gamma(x)-\int_{X} F(\gamma, x) \pi(d x) .
$$

Hence,

$$
U_{\Phi}(\delta(F))(\gamma)=\int_{X} F\left(\gamma-\epsilon_{x}, x\right) d \gamma(x)-\int_{X} F(\gamma, x) \pi(d x) .
$$

Corollary 7.4. Let $F \in \mathrm{L}$. Then $\delta(F) \in(\mathscr{S})_{-p}^{-1}$ for some $p \in \mathrm{N}$, and

$$
\mathscr{S}(\delta(F))(\xi)=\int_{X}(\mathscr{S}(F(x))(\xi) \cdot \xi(x)) \pi(d x),
$$

for all $\xi \in U_{p}$.

Proof. By equation (7.2), we have that $D_{x} \widetilde{e}(\xi, \omega)=\widetilde{e}(\xi, \omega) \xi(x)$. Hence, by Theorem 7.3 ,

$$
\begin{aligned}
\mathscr{S}(\delta(F))(\xi) & =\langle\langle\delta(F), \widetilde{e}(\xi)\rangle\rangle \\
& =\int_{X}\langle\langle F(x), \widetilde{e}(\xi) \xi(x)\rangle \pi(d x) \\
& =\int_{X} \mathscr{S}(F(x))(\xi) \cdot \xi(x) \pi(d x) .
\end{aligned}
$$


REMARK 7.5. Corollary 7.4 provides a powerful tool for solving certain types of stochastic differential equations by $\mathscr{S}$-transforming the stochastic differential equations into deterministic differential functional equations.

Define the process $W: X \times \Omega \rightarrow \mathrm{R}$ by

$$
W(x)(\omega):=\left\langle C_{1}(\omega), \epsilon_{x}\right\rangle,
$$

where $\epsilon_{x}$ denotes the Dirac delta function with mass at the point $x \in X$. We have the following relationship between the Skorohod integral, the Wick product and the Lebesgue integral.

Proposition 7.6. For all $F \in \mathrm{L}$,

$$
\delta(F)=\int_{X}(F(x) \diamond W(x)) d \lambda^{\times(d+1)}(x) .
$$

ProOF. By Corollary 7.4 and the definition of the Wick product,

$$
\begin{aligned}
\mathscr{S}\left(\int_{X} F(x) \diamond W(x) d \lambda^{\times(d+1)}(x)\right)(\xi) \\
\quad=\int_{X} \mathscr{S}(F(x) \diamond W(x))(\xi) d \lambda^{\times(d+1)}(x) \\
=\int_{X} \mathscr{S}(F(x))(\xi) \cdot \mathscr{S}(W(x))(\xi) d \lambda^{\times(d+1)}(x) \\
\quad=\int_{X} \mathscr{S}(F(x))(\xi) \cdot\left(\epsilon_{x}, \xi\right)_{\pi} d \lambda^{\times(d+1)}(x) \\
=\int_{X} \mathscr{S}(F(x))(\xi) \cdot \xi(x)(1 \otimes \dot{v})(x) d \lambda^{\times(d+1)}(x) \\
=\int_{X} \mathscr{S}(F(x))(\xi) \cdot \xi(x) \pi(d x) \\
=\mathscr{S}(\delta(F))(\xi) .
\end{aligned}
$$

The result then follows from Theorem 5.3.

\section{REFERENCES}

1. Albeverio, S., Daletsky, Y. L., Kondratiev, Y. G., and Streit, L., Non-Gaussian infinite dimensional analysis, J. Funct. Anal. 138 (1996), 311-350.

2. Benth, F. E., and Løkka, A., Anticipative calculus for Lévy processes and stochastic differential equations.

3. Cramer, H., Mathematical Methods in Statistics, Princeton University Press, Princeton, 1946. 
4. Dineen, S., Complex Analysis in Locally Convex Spaces, North-Holland Math. Stud. 57 (1981).

5. Folland, G. B., Real Analysis, John Wiley and Sons, 1984.

6. Gel'fand, I. M., Graev, M. J., and Vershik, A. M., Representations of the group of diffeomorphisms, Russian Math. Surveys 30 (1975), 3-50.

7. Gel'fand, I. M., and Vilenkin, N. Y., Generalized Functions, 4, Some Applications of Harmonic Analysis, Academic Press, New York, 1964.

8. Hida, T., Kuo, H.-H, Potthoff, J., and Streit, L., White Noise, Kluwer, Dordrecht, 1993.

9. Itô, K., Spectral type of the shift transformation of differential processes with stationary increments, Trans. Amer. Math. Soc. 81 (1956), 253-263.

10. Ito, Y., and Kubo, I., Calculus on Gaussian and Poisson white noises, Nagoya Math. J. 111 (1988), 41-84.

11. Kachanovsky, N. A., On biorthogonal approach to a construction of non-Gaussian analysis and application to the Poisson analysis on the configuration space, Methods Funct. Anal. Topology 6 (2) (2000), 13-21.

12. Kondratiev, Y. G., Da Silva, J. L., and Streit, L., Generalized Appell systems, Methods Funct. Anal. Topology 3 (3) (1997).

13. Kondratiev, Yu., Da Silva, J. L., L. Streit and Us, G., Analysis on Poisson and gamma spaces, Infin. Dimens. Anal. Quantum Probab. Relat. Top. 1 (1) (1998), 91-117.

14. Lee, Y-J, and Shih, H-H, Donsker's delta function of Lévy processes, Acta Appl. Math. 63 (2000), 219-231.

15. Matthes, K., Kerstan, J., and Mecke, J., Infinitely Divisible Point Processes, Wiley Series in Prob. and Math. Stat. (1978).

16. Nachbin, L., Topology on Spaces of Holomorphic Mappings, Springer-Verlag, 1969.

17. Nualart, D., and Vives, J., Anticipative calculus for the Poisson process based on Fock space, Séminaire de Probabilités XXIV, 154-165, Lecture Notes in Math. 1426 (1988/89).

18. Protter, P., Stochastic Integration and Differential Equations, Springer-Verlag, 1990.

19. Reed, M., and Simon, B., Methods of Modern Mathematical Physics; functional analysis, Academic Press, 1972.

20. Schaefer, H. H., Topological Vector Spaces, Springer, New York, 1971.

21. Us, G. F., Dual Appell Systems in Poissonian Analysis, Methods Funct. Anal. Topol. 1 (1995), 93-108.

DEPARTMENT OF MATHEMATICS

UNIVERSITY OF OSLO

P.O. BOX 1053, BLINDERN

N-0316 OSLO

NORWAY

E-mail: alokka@math.uio.no,proske@math.uio.no 